\title{
An Efficient Iris Coding Based on Gauss-Laguerre Wavelets
}

\author{
H. Ahmadi ${ }^{1,2}$, A. Pousaberi ${ }^{1}$, A. Azizzadeh ${ }^{3}$, and M. Kamarei ${ }^{1}$ \\ ${ }^{1}$ Dept. of Electrical and Computer Engineering,University of Tehran \\ ${ }^{2}$ Dept. of Electrical and Computer Engineering, University of British Columbia \\ ${ }^{3}$ Research Center, Ministry of Communication, Tehran, Iran \\ noubari@ece.ubc.ca, a.poursaberi@ece.ut.ac.ir, azad@itrc.ac.ir, \\ kamarei@ut.ac.ir
}

\begin{abstract}
In this paper preliminary results of a new iris recognition algorithm using Gauss-Laguerre filter of circular harmonic wavelets are presented. Circular harmonic wavelets (CHWs) applied in this paper for iris pattern extraction, are polar-separable wavelets with harmonic angular shape. The main focus of this paper is on iris coding using Gauss-Laguerre CHWs which constitute a family of orthogonal functions satisfying wavelet admissibility condition required for multiresolution pyramid structure. It is shown that GaussLaguerre wavelets having rich frequency extraction capabilities are powerful tools for coding of iris patterns. By judicious tuning of Laguerre parameters, a 256-byte binary code is generated for each iris. A fast matching scheme based on Hamming distance is used to compute the similarity between pairs of iris codes. Preliminary experimental results on CASIA and our database indicate that the performance of the proposed method is highly accurate with zero false rate and is comparable with Daugman iris recognition algorithm well publisized in literature.
\end{abstract}

Keywords: Biometrics, Iris recognition, Gauss-Laguerre wavelets, Circular harmonic wavelets.

\section{Introduction}

Security and surveillance of information and personnel is becoming more and more important recently, in part due to the rapid development of information technology (IT) and its wide spread applications in all aspects of daily life. For surveillance of personnel, biometrics as a science of personal identification that utilize physical/biological or behavioral characteristics of an individual, are now widely used in numerous security applications. For a biometric system to be a valid candidate for use in human identification, it is required that it embodies any number of unique characteristics of an individual that remain consistent during the life of a person. Fingerprints, voiceprints, retinal blood vessel patterns, face, iris pattern, handwriting are examples of biometrics that can be used in place of non-biometric methods. Irisbased biometrics is considered to provide a high accuracy personal identification mainly due to rich texture of iris pattern as well as nonintrusive nature of biometric extraction [1]. 
Iris recognition is based on the visible features of the human iris (see Fig. 1) that include rings, furrows, freckles, and the iris corona. These unique features are protected by the body's own mechanisms and can not be modified without risk. As such iris features are considered to be the highly accurate and reliable biofeatures for personal identification [1] and has been a subject of wide attentions during the last decade. Standard iris recognition process consists of the following three major steps.

- Preprocessing step which includes image capturing, image filtering and enhancement (optional) followed by iris localization, normalization, denoising and image enhancement.

- Iris feature extraction stage.

- Iris feature classification

Several algorithms have been proposed by numerous groups for iris-based biometrics of which the most publisized and accepted algorithm, belongs to John Daugman [2]. Daugman used multiscale quadrature wavelets to extract phase structure information of the iris texture and generate a 2048-bit iris code. For identification the difference between a pair of iris codes is evaluated using Hamming distance criteria. In Daugman scheme, it has been shown that a Hamming distance of less than 0.34 measured with any of iris templates in database was acceptable for identification. Since introduction of Daugman algorithm, numerous attempts have been made by other groups to arrive at compatible or alternative algorithms and several claims have been made on the adequacy of these algorithms for iris recognition. Daugman used the following three innovations for feature extraction and subsequent coding:

1. An Integro-differential scheme for iris segmentation.

2. A Cartesian to polar transformation followed by normalization.

3. A 256 binary code based on Gabor filters.

These three steps have also been utilized by other research groups in which alternative algorithms including modification of Gabor filters have been proposed. Among them, algorithms proposed by Wildes [3], Ma [4] are claimed to be comparable with Daugman scheme in accuracy of recognition. In this paper, we present an alternative iris coding scheme which utilizes Gaussian-Laguerre filters during iris coding where it is shown that results are commensurable with those of Daugman. The proposed algorithm using Gaussian-Laguerre wavelets first introduced in this paper was tested using CASIA data base as well as data generated by this research group. The rich information of Gaussian Laguerre filter in various frequency bands enables an extraction of the necessary iris patterns in an effective manner and generates a meaningful unique code for each individual. The proposed algorithm includes also an algorithm based on Radon transformation for iris localization which is computationally efficient and contributes significantly to increase the speed of iris localization. In the sequel, a brief reference is made to some of the related research works on iris-based biometrics followed with an overview of our proposed algorithm in Section 2 . Description of circular harmonic wavelets (CHW) is included in Section 3. Image preprocessing for segmentation, feature extraction and pattern matching are 

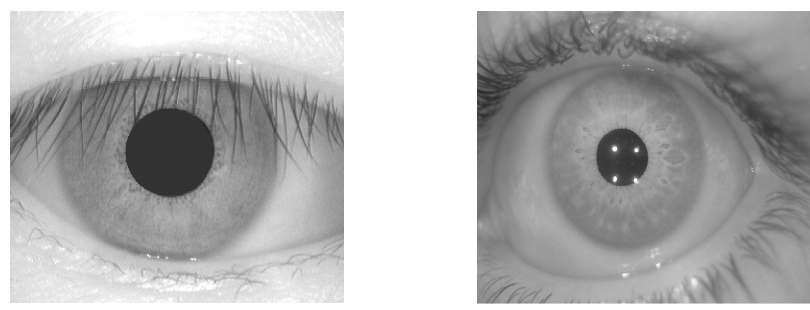

Fig. 1. Typical iris images, (left) CASIA data base, (right) Our captured iris image

given in Sections 4 and 5, respectively. Experimental results on Iris databases are reported in Section 6. Further works are described in Section 7. Finally Section 7 concludes this paper.

\section{A Brief Overview of Literature and Proposed Scheme}

Biometrics using iris features is relatively new with most of the works done during last decade. A pioneering work was done by John Daugman [2] in which multiscale quadrature wavelets were used to extract texture phase structure information of the iris to generate a 2,048-bit iris code. Euclidean distance was used to evaluate the difference between a pair of iris representations. It was shown that, a Hamming distance of lower than 0.34 with any of iris templates in database will suffice for identification. Ma et al.[4],[5] adopted a well-known texture analysis method (multichannel Gabor filtering) to capture both global and local details in an iris image. They studied Gabor filter families for feature extraction. Wildes et al. [3] considered Laplacian pyramid constructed in four different resolution levels and used their normalized correlation for matching Boles and Boashash [6] used a zero-crossing of $1 \mathrm{D}$ wavelet at various resolution levels to distinguish the texture of the iris. Tisse et al. [7] constructed the analytic image (a combination of the original image and its Hilbert transform) to demodulate the iris texture. Lim et al. [8] used 2D Haar wavelet and quantized the 4th level high frequency information to form an 87-binary code length as feature vector and applied a LVQ neural network for classification. Woo Nam et al. [9] exploited a scale-space filtering to extract unique features that uses the direction of concavity of image from an iris image. A modified Haralick's cooccurrence method with multilayer perceptron has also been introduced for extraction and classification of the iris images [10]. In a prior work carried-out by this group Daubechies2 wavelets were utilized for the analysis of local patterns of iris [11] [12].

A typical automatic iris recognition system (AIRS) requires an implementation of several steps as indicated in Fig. 2. At first step, an imaging system must be designed to capture a sequence of iris images with sufficient details from the subject. After image capturing, image preprocessing is to be applied on the images which includes several important steps stages consisting of identification of boundaries of iris, image enhancement, normalization and coordinate transformation. To implement an automatic iris recognition system (AIRS), we used a new algorithm for iris segmentation that is considered to perform faster than the currently reported methods. This was followed by a new iris feature extraction and coding algorithm using 
Laguerre Gaussian wavelets. For matching process as the last step in identification process and for comparing iris code with database, Hamming distance between class of each iris code and input code was utilized. Descriptions of details of proposed algorithms are given in sections that follow.

\section{Gaussian Laguerre Wavelets}

The circular harmonic wavelets (CHWs) are polar-separable wavelets, with harmonic angular shape. They are steerable in any desired direction by simple multiplication with a complex steering factor and as such they are referred to self-steerable wavelets. The CHWs were first introduced in [14] which utilizes concepts from circular harmonic functions (CHFs) employed in optical correlations for rotation invariant pattern recognition. The same functions also appear in harmonic tomographic decomposition and have been considered for the analysis of local image symmetry. In addition; recently, CHFs have been employed for the definition of rotation-invariant pattern signatures [8]. A family of orthogonal $\mathrm{CHWs}$ forming a multiresolution pyramid referred to as circular harmonic pyramid (CHP) is utilized for coefficient generation and coding. In essence, each CHW pertaining to the pyramid represents the image by translated, dilated and rotated versions of a CHF. At the same time, for a fixed resolution, the CHP orthogonal system provides a local representation of the given image around a point in terms of CHFs. The self-steerability of each component of the CHP can be exploited for pattern analysis in presence of rotation (other than translation and dilation) and, in particular, for pattern recognition, irrespective of orientation [15].

CHFs are complex, polar separable filters characterized by harmonic angular shape, a useful property to build rotationally invariant descriptors. A scale parameter is also introduced to perform a multiresolution analysis. The Gauss-Laguerre filters (CHFs) which constitute a family of orthogonal functions satisfying wavelet

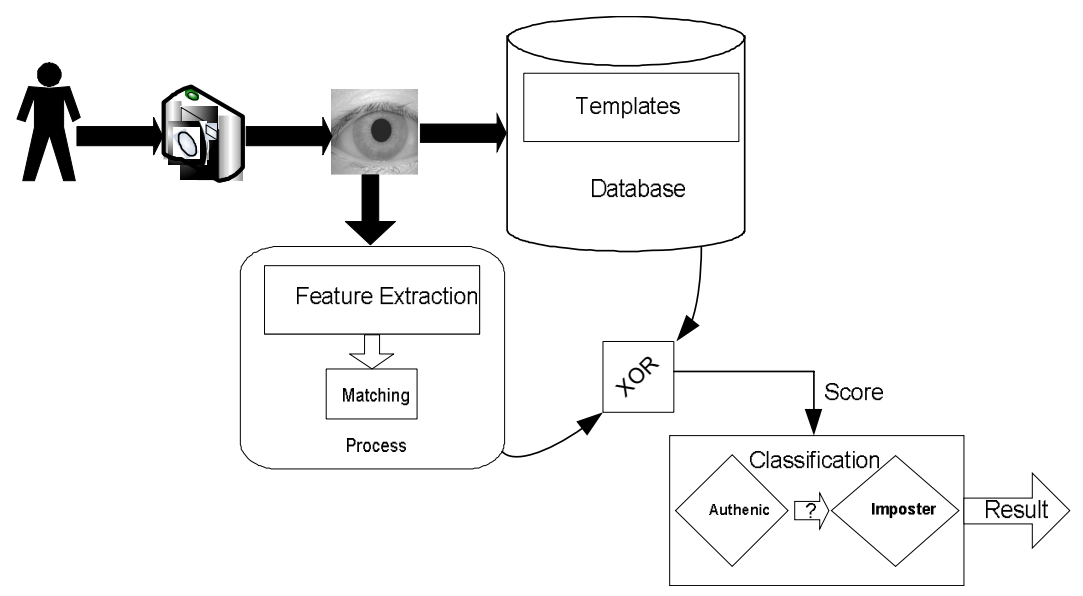

Fig. 2. Flow diagram of system for eye localization and iris segmentation 
admissibility condition required for multiresolution wavelet pyramid analysis are used. Similar to Gabor wavelets, any image may be represented by translated, dilated and rotated replicas of Gauss-Laguerre function. For a fixed resolution, the GaussLaguerre CHFs provide a local representation of the image in the polar coordinates system centered at a given point, named pivot. This representation is called the GaussLaguerre transform [16]. A Circular harmonic function is a complex polar separable filter characterized by a harmonic angular shape, which is represented in polar coordinates as:

$$
L_{k}^{(n)}(r, \theta)=h(r) \cdot e^{i n \theta}
$$

where $h(r)$ is:

$$
h(r)=(-1)^{k} 2^{\frac{(|n|+1)}{2}} \pi^{\frac{|n|}{2}}\left[\frac{k !}{(|n|+k) !}\right]^{\frac{1}{2}} r^{|n|} L_{k}^{n}\left(2 \pi r^{2}\right) e^{-\pi r^{2}}
$$

Here $r$ and $\theta$ are polar coordinates and $L_{k}^{(n)}($.$) is the generalized Laguerre polyno-$ mial as follows:

$$
L_{k}^{n}(r)=\sum_{h=0}^{k}(-1)^{k}\left(\begin{array}{l}
n+k \\
k-h
\end{array}\right) \frac{r^{h}}{h !}
$$

For different radial order $k$ and angular $\operatorname{order} n$, these functions called GaussLaguerre (GL) functions and from an orthogonal basis set under the Gaussian function. As any CHF, GL functions are self-steering, i.e. they rotate by an angle $\phi$ when multiplied by factor $e^{j n \phi}$. In particular, the real and imaginary part of each GL function form a geometrically in phase-quadrature pair. Moreover, GL functions are isomorphous with their Fourier transform. It is shown [15] that each GL function defines an admissible dyadic wavelet. Thus, the redundant set of wavelets corresponding to different GL functions constitutes a self-steering pyramid, useful for local and multiscale image analysis. The real part of GL function is depicted in Fig. 3. For better visualization, the resolution of filter is enhanced. GL pyramid (real part CHW) is also shown in Fig. 4.

An important feature of GL functions as applied to iris recognition system is that GL function with various degrees of freedom can be tuned to significant visual features. For examples, for $n=1$, GLS are tuned to edges, for $n=2$ to ridges, for $n=3$ to equiangular forks, for $n=4$ to orthogonal crosses, irrespective of their actual orientation. Let $I(x, y)$ be the observed image. For every site of the plane, it is possible to perform the GL analysis by convolving it with each properly scaled GL function,

$$
g_{j n k}(x, y)=\frac{1}{a 2^{j}} L_{k}^{n}\left(\frac{r \cos \theta}{a 2^{j}}, \frac{r \sin \theta}{a 2^{j}}\right)
$$

where $a 2^{j}$ are the dyadic scale factors. 


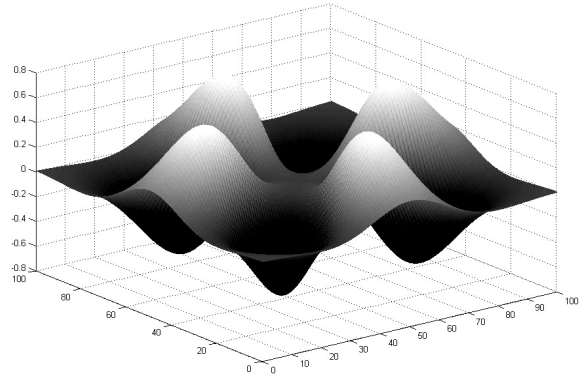

Fig. 3. Real part of GL function for $n=4, k=0, j=2$

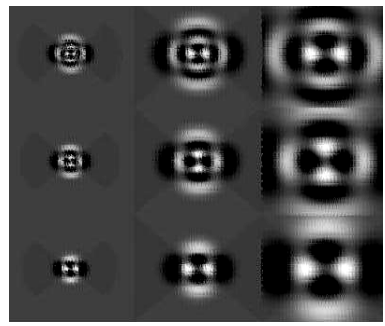

Fig. 4. GL pyramid. Real part of CHW for $n=2, k=0,1,2, j=0,1,2$.

\section{Image Preprocessing}

Preprocessing in iris recognition consists of three steps: iris edge detection, normalization and enhancement. A captured image contains not only the iris but also contains segments of eyelid, eyelash, pupil and sclera that all are considered to be not desirable. Distance between camera and eye, environment light conditions (dilation of pupil) can influence the size of iris. Therefore, image preprocessing is a necessary step before any feature extraction step can be applied to overcome these problems. However, in the proposed algorithm due to its robustness against illumination and contrast effects, we have not considered enhancement or noise removal during iris preprocessing.

\subsection{Iris Localization}

Iris boundaries are considered to be composed of two non concentric circles. For iris recognition, it is necessary to determine inner and outer boundaries, radius and centers. Several approaches have been proposed using iris edge detection. Our method is based on iris localization using Radon transform and locally searching for iris image. The details of the approach are in [13]. The key contribution of the Radon transformation and consequent local search approach is its fast response in identifying the iris boundaries. For instance, time of detection on CASIA image database [17] was only .032 seconds in average evaluated on a platform of 2.2 Intel CPU, $512 \mathrm{M}$ 
RAM and MATLAB source code. In comparison, our approach exhibited a high order of success rate and time consuming between existed methods. The results of detection on two databases [13], show high order of reliability on iris segmentation.

\subsection{Iris Normalization}

Different image acquisition process and conditions can influence the results of identification. The dimensional incongruities between eye images are mostly due to the stretching of the iris caused by pupil expansion/ contraction from variation of the illuminations. Other factors that contribute to such deformations include variance of camera and eye distance, rotation of the camera or head. Hence a solution must be contrived to remove these deformations. The normalization process, projects iris region into a constant dimensional ribbon so that two images of the same iris under different conditions have characteristic features at the same spatial location. Daugman [2] suggested a normal Cartesian to Polar transform that remaps each pixel in iris area into a pair of polar coordinates $(r, \theta)$ where $r$ and $\theta$ is on the interval $\left[\begin{array}{ll}0 & 1\end{array}\right]$ and $\left[\begin{array}{ll}0 & 2 \pi\end{array}\right]$ respectively. In this paper, we have used Daugman's approach on the images of size of $512 \times 128$ and $12.5 \%$ of lower part of the normalized image was discarded.

\section{Feature Extraction}

As stated earlier, GL functions provide self steering pyramidal analysis structure that can be used to extract iris features and for iris coding efficiently. Using GL functions where by proper choice of their parameters, it is possible to generate a set of redundant wavelets that enable an accurate extraction of complex texture features of an iris. Redundancy of wavelet transform and a higher degree of freedom in selecting parameters of GL function as compared with those of Gabor wavelets, makes GL function highly suitable for iris feature extraction. Self steering pyramid structure of LG-based image analysis is distinctly different from Gabor wavelets where by choosing the parameters of GL functions, we are able to carryout both local and multiscale image analysis of a given image in a more effective manner .To take advantage of the degrees of freedom provided by LG function, it is necessary that the parameters of the filters be tuned to significant visual features and iris texture patterns so that it can extract desirable frequency information of iris patterns. In our experiment it was found that for $n=2$, quality results were obtained where several simulations runs were carried out to verify the selection of parameters in which filters were convolved directly with the mapped iris image. Other parameters in this manner were also adjusted by trial and error and observing the simulation results. The output of filtered image is a complex value code that contains information of iris for coding. Based on the sign of each entry, we assign +1 to positive and 0 to others codes. Finally a 2048 binary bite is generated for each iris a sample of the code is depicted in Fig. 5. For matching process, Hamming distance is used. Rotation is considered here by shifting code versus templates in database. We take into account 12 bite shifts in both direction (left and right) and 2 bit shifts upwards and downwards. The minimum distance between input code and all codes in database for each class is assigned for decision making. 


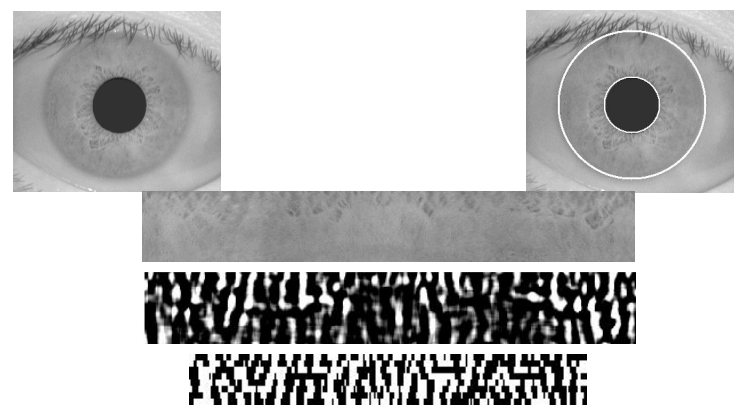

Fig. 5. Step by step of flowchart. (from left to right - up to down) input image, segmented iris, normalized iris, complex-valued iris code, binary code.

\section{Experimental Results}

To evaluate the performance of the proposed algorithm, our alghoritm was tested on two iris databases: a) CASIA Database [17] and a) set of iris image collected locally using using an iris camera system built at the research lab. Our preliminary database constructed locally has 280 images from 40 distinct subjects. Three images were taken at the first session followed by 4 images that were taken at second session. Our local iris imaging system was a modified webcam with Infrared lighting. For each iris class,

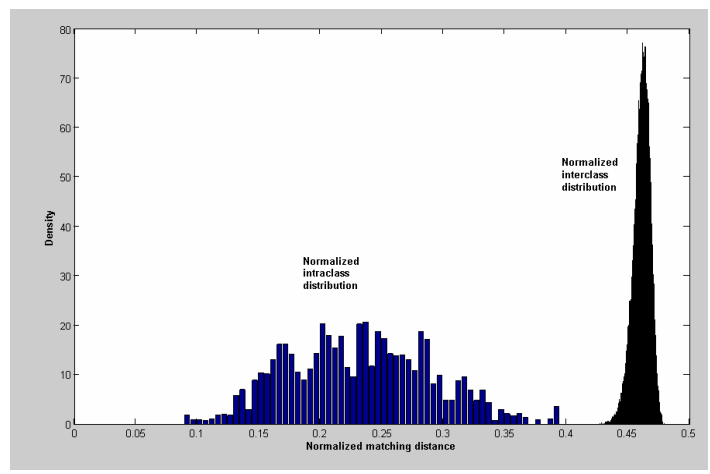

Fig. 6. The distribution of distance between authorized abd imposter users. A reasonable separation is exist between them.
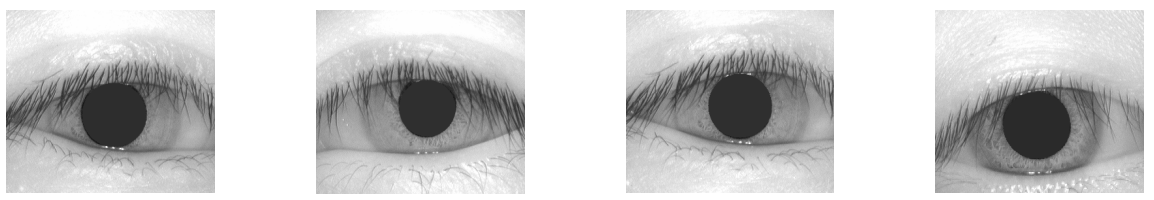

Fig. 7. Four iris images that failed in verification process. As can be seen, all are heavily occluded with eyelids and eyelashes. 
we choose three samples taken at the first session for training and all samples captured at second sessions serve as test samples. This is also consistent with the widely accepted practice for testing biometrics algorithms [18]. We tested the proposed algorithms in two modes of. identification and verification. In identification tests, correct classification rate was $100 \%$ and complete separibality between interclass and intraclass distances is achived in both databases. In verification mode, a 1:1 search for each image was done. The algoritm was able to succesfully verify each input image except in case of 4 iris images in CASIA and 0 image in our local database. The discrepency and the error can easily be attributed to poor image quality and image capturing process. Fig. 6 shows the distribution of intra-class and inter-class matching distance of algorithm. The four images that failed for correct identification, are shown in Fig. 7 and as it can be seen, images are extremely occluded or has excessive pupil dilation. The system was implemented by MATLAB, $1.8 \mathrm{GHz}$ Intel processor, $256 \mathrm{M}$ RAM. The entire time of process including localization, preprocessing and normalization, feature extraction and matching is only $(.2+.05+.15+.05=.45$ Seconds).

\section{Further Works}

This paper outlines the results of our preliminary attempt for designing an effective iris recognition system for personal identification. As such we plan modifications in the system to improve the system both in hardware and in software algorithm. In our future, we will attempt to improve identification results for which image enhancement will be incorporated during preprocessing stage. Such preprocessing is anticipated to increase classification accuracy i.e. increase of separation distance between inter and intra classes. It is also planned to examine a new scheme in XOR classification in which a circular shifting to simulate eye rotation will be considered. Masking of the eyelids and eyelashes is also planned as part of future works.

\section{Conclusion}

In this paper, a new and an efficient algorithm for fast iris recognition is proposed for which Gaussian-Laguerre (LG) Wavelets have been utilized. Gauss-Laguerre filter is conceived to be more compatible for extraction of iris texture information as compared with commonly used Gabor filter. By adjusting Laguerre parameters and applying to normalized iris, a 256-byte in binary form is generated for each individual. An Exclusive OR classifier was used to perform and evaluate matching score. Despite of the absence of iris enhancement and denoising during image preprocessing, the results showed that the algorithm is sufficiently robust against contrast and illumination factors. Experimental results on a set of two databases indicated superior performance of the LG based algorithm in both identification and verification modes.

\section{References}

1. Jain, A., Bolle, R., Pankanti, S.: Biometrics: Personal Identification in a Networked Society. Kluwer Academic Publishers, Dordrecht (1999)

2. Daugman, J.: High Confidence Visual Recognition of Persons by a Test of Statistical Independence. IEEE Trans. PAMI 15, 1048-1061 (1993) 
3. Wildes, R.P.: Iris Recognition: An Emerging Biometric Technology. In: Proc. IEEE, vol. 85, pp. 1348-1363 (1997)

4. Ma, L., Tan, T., Wang, Y., Zhang, D.: Efficient Iris Recognition by Characterizing Key Local Variations. IEEE Trans. Image Processing 13 (2004)

5. Ma, L., Wang, Y., Tan, T.: Personal Iris Recognition Based on Multichannel Gabor Filtering. In: ACCV2002, Melbourne Australia (2002)

6. Boles, W., Boashash, B.: A Human Identification Technique Using Images of the Iris and Wavelet Transform. IEEE Trans. Signal Processing 46, 1085-1088 (1998)

7. Tisse, C., Martin, L., Torres, L., Robert, M.: Person Identification Technique Using Human Iris Recognition. In: Proc. Vision Interface, pp. 294-299 (2002)

8. Lim, S., Lee, K., Byeon, O., Kim, T.: Efficient Iris Recognition through Improvement of Feature Vector and Classifier. ETRI Journal 23 (2001)

9. Nam, K.W., Yoon, K.L., Bark, J.S., Yang, W.S.: A Feature Extraction Method for Binary Iris Code Construction. In: Proc. 2nd Int. Conf. Information Technology for Application (2004)

10. Jaboski, P., Szewczyk, R., Kulesza, Z.: Automatic People Identification on the Basis of Iris Pattern Image Processing and Preliminary Analysis. In: Proc. Int. Conf. on Microelectronics, Yugoslavia, vol. 2, pp. 687-690 (2002)

11. Poursaberi, A., Araabi, B.N.: A Half-Eye Wavelet Based Method for Iris Recognition. In: Proc. 5th Int. Conf. Intelligent Systems Design and Applications (ISDA), Wroclaw Poland (2005)

12. Poursaberi, A., Araabi, B.N.: Iris Recognition for Partially Occluded Images: Methodology and Sensitivity Analysis. J. Applied Signal Processing (to be published in February 2006)

13. Torkamani, A., Azizzadeh, A.: Iris Detection as Human Identification. J. IET Image Processing Journal (to be published 2007)

14. Jacovitti, G., Neri, A.: Multiscale Image Features Analysis with Circular Harmonic Wavelets. In: Proc. SPIE 2569, Wavelets Appl. Signal Image Process, vol. 2569, pp. 363$372(1995)$

15. Jacovitti, G., Neri, A.: Multiresolution Circular Harmonic Decomposition. IEEE Trans. Signal Processing 48, 3242-3247 (2000)

16. Capdiferro, L., Casieri, V., Jacovitti, G.: Multiple Feature Based Multiscale Image Enhancement. In: Proc. IEEE DSP. IEEE Computer Society Press, Los Alamitos (2002)

17. www.sinobiometrics.com

18. Mansfield, T., Kelly, G., Chandler, D., Kane, J.: Biometric Product Testing Final Report. In: issue 1.0, Nat'1 Physical Laboratory of UK (2001) 